\title{
Alpha and gamma band oscillations index differential processing of acoustically reduced and full forms
}

\author{
Linda Drijvers ${ }^{\mathrm{a}, \mathrm{b}, *}$, Kimberley Mulder ${ }^{\mathrm{a}}$, Mirjam Ernestus ${ }^{\mathrm{a}, \mathrm{c}}$ \\ ${ }^{a}$ Radboud University, Centre for Language Studies, Erasmusplein 1, 6525 HT Nijmegen, The Netherlands \\ ${ }^{\mathrm{b}}$ Radboud University, Donders Institute for Brain, Cognition, and Behaviour, Montessorilaan 3, 6525 HR Nijmegen, The Netherlands \\ 'Max Planck Institute for Psycholinguistics, Wundtlaan 1, 6525 XD Nijmegen, The Netherlands
}

\section{A R T I C L E I N F O}

Article history:

Received 18 May 2015

Revised 4 January 2016

Accepted 23 January 2016

\section{Keywords:}

Speech comprehension

Neuronal oscillations

Speech reduction

Cognitive load

Semantic integration

\begin{abstract}
A B S T R A C T
Reduced forms like yeshay for yesterday often occur in conversations. Previous behavioral research reported a processing advantage for full over reduced forms. The present study investigated whether this processing advantage is reflected in a modulation of alpha $(8-12 \mathrm{~Hz})$ and gamma $(30+\mathrm{Hz})$ band activity. In three electrophysiological experiments, participants listened to full and reduced forms in isolation (Experiment 1), sentence-final position (Experiment 2), or mid-sentence position (Experiment 3). Alpha power was larger in response to reduced forms than to full forms, but only in Experiments 1 and 2 . We interpret these increases in alpha power as reflections of higher auditory cognitive load. In all experiments, gamma power only increased in response to full forms, which we interpret as showing that lexical activation spreads more quickly through the semantic network for full than for reduced forms. These results confirm a processing advantage for full forms, especially in non-medial sentence position.
\end{abstract}

(c) 2016 Elsevier Inc. All rights reserved.

\section{Introduction}

Natural, conversational speech is characterized by an incredible amount of variation in the pronunciation of words. One of the reasons for this variation is that speakers reduce words in casual speech by producing words with fewer or altered segments (for an overview, see Ernestus \& Warner, 2011). For instance, in conversational English, words like yesterday and hilarious are often reduced to

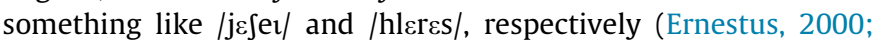
Johnson, 2004). Native listeners seem to understand reduced word forms effortlessly in connected speech. This paper contributes to the research on how native listeners understand these forms.

So far, the processing of reduced word forms has been addressed in several corpus-based studies and in several behavioral production and comprehension studies. One of the most consistent findings is that reduced forms are processed less easily than full forms. Listeners need more time and more linguistic context to comprehend reduced word forms than full forms (e.g. Ernestus \& Baayen, 2007; Ernestus, Baayen, \& Schreuder, 2002; Janse, Nooteboom, \& Quene, 2007; Tucker \& Warner, 2007; Ernestus,

* Corresponding author at: Radboud University, Centre for Language Studies, Donders Institute for Brain, Cognition and Behaviour, Wundtlaan 1, 6525 XD Nijmegen, The Netherlands.

E-mail address: linda.drijvers@mpi.nl (L. Drijvers).
2014) and speakers need more time to name objects if they are instructed to produce reduced forms rather than full forms (Bürki, Ernestus, \& Frauenfelder, 2010).

After listeners have identified a reduced word form, it also seems to take longer before the corresponding semantic network is activated than when the word is pronounced in full. Van de Ven, Tucker, and Ernestus (2011) performed a lexical decision experiment in which consecutive stimuli functioned as primes and targets and prime-target pairs differed in their semantic relatedness. The researchers only analyzed those targets that were correctly classified as real words and were preceded by primes that were also correctly classified as real words. They observed a clear difference between responses to targets that were primed by full forms and responses to targets that were primed by reduced forms. If the targets were presented $1000 \mathrm{~ms}$ after the responses to the primes, only the primes that were fully articulated produced priming effects. However, if the targets were presented 1500-1600 ms after the responses to the primes, both full and reduced forms produced priming. These results strongly suggest that reduced forms may take longer than full forms to activate their semantic networks after they have been identified.

In all studies reporting a processing advantage for full forms over reduced forms, the words were presented in isolation (e.g. Ernestus et al., 2002; Janse et al., 2007; Ranbom \& Connine, 2007; Tucker \& Warner, 2007) or at the end of simple sentences 
(e.g. Bürki et al., 2010; Ranbom \& Connine, 2007). This raises the question whether full forms still have a processing advantage over reduced forms if these forms occur in the middle of full sentences, that is, in the position in which they occur most frequently (e.g. Pluymaekers, Ernestus, \& Baayen, 2005).

There are several reasons for why we may expect that the processing advantage for full forms disappears in connected speech. First, it seems counter-intuitive that word forms that are highly frequent are hard to process. Second, Bürki et al. (2010) showed that full forms lose some of their special status when they are preceded by possessive pronouns instead of just definite articles. Finally, a few off-line behavioral studies show that listeners rely on all types of cues in the context, ranging from subtle phonetic cues (e.g. Janse \& Ernestus, 2011) to semantic information (e.g. Van de Ven, Ernestus, \& Schreuder, 2012). If this information is available, as is the case in natural conversations, listeners may process reduced forms as easily as full forms.

This hypothesis is difficult to test with behavioral experimental paradigms. In many experimental paradigms, including the lexical decision task, words are presented in isolation or at the end of simple sentences. In tasks in which words can occur in the middle of sentences, like identity cross-modal priming, the words are presented together with their orthographic transcriptions, which typically correspond to and therefore introduce a bias towards the full forms. Finally, an experimental task like the visual world paradigm severely restricts the possible words that can be tested because the words have to be depictable.

The present study investigates the processing of full and reduced pronunciation variants by analyzing neuronal oscillations induced by these forms. We investigate whether there are qualitative and quantitative differences in the alpha and gamma bands for full and reduced forms, and whether these differences are modulated by linguistic context. To our knowledge, no other study has compared the oscillations involved in the processing of full and reduced speech.

Neuronal oscillations predominantly reflect the extent to which the underlying neuronal activity synchronizes in the brain. These changes in oscillatory synchronization are thought to provide a window into the dynamics of the coupling and uncoupling of networks involved in cognitive processing (e.g. Hipp, Engel, \& Siegel, 2011; Bastiaansen, Mazaheri, \& Jensen, 2012; Varela, Lachaux, Rodriguez, \& Martinerie, 2001). By studying oscillatory dynamics, we aim to gain insight into whether different processes are involved in the comprehension of full and reduced forms, in different contexts.

Alpha oscillations $(8-12 \mathrm{~Hz})$ are thought to reflect a mechanism that functionally inhibits task-irrelevant brain areas or distracting neural activity (Foxe \& Snyder, 2011; Jensen \& Mazaheri, 2010; Mazaheri et al., 2014), whereas suppression of alpha activity seems to remove this inhibitory gating and allows for higher frequencies (e.g., gamma oscillations) to occur.

Functional inhibition has been observed in tasks involving working memory and selective attention in the (audio)visual domain, but also in studies on language comprehension, such as studies on degraded speech processing (e.g. Adrian, 1944; Foxe, Ca, \& Ahlfors, 1998; Fu et al., 2001; Jensen \& Mazaheri, 2010; Klimesch, Sauseng, \& Hanslmayr, 2007; Obleser \& Weisz, 2012; Obleser, Wöstmann, Hellbernd, Wilsch, \& Maess, 2012; Strauß, Kotz, Scharinger, \& Obleser, 2014). For example, in the audiovisual domain, Foxe et al. (1998) reported a $10-\mathrm{Hz}$ parietal-occipital enhancement over visual areas when participants were cued to attend to an auditory stimulus (a beep) instead of a visual stimulus (a flash). In preparation for the anticipated and attentionally more relevant auditory input, the visual attentional system was functionally inhibited to allocate attentional resources to the auditory modality.
Recent studies on degraded speech processing have argued that the suppression of alpha oscillations is associated with more effective language processing whereas alpha power remains high when the language processing network is inhibited (Obleser \& Kotz, 2010, 2011; Obleser \& Weisz, 2012; Obleser et al., 2012; Strauß, Kotz, et al., 2014). The lack of alpha suppression that arises after participants listened to severely degraded speech could reflect neural oscillators that keep alpha power high to rule out erroneous activations in relevant language- and meaning-related areas (Obleser \& Weisz, 2012) and is mostly observed after a full linguistic utterance (Klimesch et al., 2007; Shahin, Picton, \& Miller, 2009). Another recent study demonstrated a parametric suppression in alpha band activity as items increasingly matched real words, with lowered functional inhibition for more word-like input (Strauß, Kotz, et al., 2014). Strauß and colleagues propose that the observed enhanced alpha power seems to 'gate' words towards lexical integration and alpha oscillations can be seen as an indicator of cognitive load in audition (Strauß, Wostmann, \& Obleser, 2014). Thus, alpha oscillations could provide a window into whether reduced form processing causes more auditory cognitive load than full form processing.

As the behavioral literature shows that reduced forms are harder to process than full forms, we expect that alpha power will increase more during reduced form processing than during full form processing. This larger increase in alpha power in response to reduced forms could reflect the listener's greater need for functional inhibition towards integration with the appropriate meaning representation and more effortful speech processing, resulting in a higher auditory cognitive load compared to full form processing. Since we assume that reduced forms are more easily understood in sentence medial context than in isolation or in sentence final position, we expect that alpha power will increase the least for reduced words presented in mid-sentence position.

In contrast to alpha oscillations, gamma oscillations (30$100 \mathrm{~Hz}$ ) are related to the maintenance of working memory representations and active cognitive processing (e.g. Jokisch \& Jensen, 2007). In language comprehension studies, gamma power increases have been associated with semantic unification operations such as the integration of the meaning of an incoming word in a given speech context, access to the mental lexicon (Bastiaansen et al., 2012), the activation of local functional networks supporting semantic representations (Mellem, Friedman, \& Medvedev, 2013) and the predictability of an upcoming word based on the preceding sentence context (Wang, Zhu, \& Bastiaansen, 2012).

For instance, gamma power increases were observed in response to words that were semantically appropriate in a given sentence context, but not when the word induced a semantic violation in the sentence (Hald, Bastiaansen, \& Hagoort, 2006), and when participants were presented with sentences in their native language, but not when they were presented with sentences in phonologically related or unrelated languages (Peña \& Melloni, 2012). Finally, an increase in gamma power was observed when participants had successfully comprehended a degraded speech signal (Hannemann, Obleser, \& Eulitz, 2007). In this latter study, the increase in gamma power has been taken to indicate successful matching of degraded speech to lexical memory traces.

On the basis of these studies on gamma band power and the behavioral study by Van de Ven et al. (2011), we can formulate predictions about how gamma band power may differ between when listeners hear reduced versus full forms. Van de Ven and colleagues showed that reduced words only prime semantically related words if the time-interval between the two words is not very short. This suggests that reduced forms take longer to activate their semantic networks. If this interpretation is correct, we expect that gamma power increases are smaller, delayed or even absent for reduced 
forms compared to full forms. This could indicate that the activation of semantic representations might be delayed for reduced forms. Possibly, the difference between full and reduced forms is smaller if the words occur in mid-sentence position rather than in isolation because reduced forms occur more frequently in mid-sentence position.

In this paper, we investigate whether and how oscillatory activity in the alpha and gamma bands is modulated during listening to reduced and full forms. The reduction phenomenon under investigation is schwa reduction in Dutch. Schwa is a mid-central vowel that often occurs in unstressed syllables (e.g. 'u' in support). In Dutch, schwa is often very short or completely absent (e.g. Pluymaekers et al., 2005; Schuppler, Ernestus, Scharenborg, \& Boves, 2011; Van Bergem, 1994). We focus on schwa reduction in the highly frequent Dutch prefixes be- /bo/, ver- / vo(r)/ and ge- / $\mathrm{x} ə /$ of verb forms.

We investigate the processing of full and schwa reduced verb forms in three speech contexts: in isolation (Experiment 1), in sentence-final position (Experiment 2) and in mid-sentence position (Experiment 3).

\section{Experiment 1: words in isolation}

\subsection{Methods}

\subsubsection{Participants}

All participants tested in this study were native speakers of Dutch, were paid to participate, participated only in one experiment, and reported normal hearing, no language impairments, no motor disabilities or neurological impairments and had normal or corrected-to-normal vision. For Experiment 1, we tested thirty-five right-handed participants (Mean age $=21.3, \mathrm{SD}=2.7$ ) Five participants were excluded from further analysis because of excessive artifacts in the EEG recording (3) or technical failures (2).

\subsubsection{Materials}

We selected 80 Dutch infinitives starting with the unstressed prefixes be- (/bə/, e.g. bevallen /bə'valə/ 'to give birth'), ge- (/хә/, e.g. gedragen /хә'draxə/ 'to act'), or ver- (/vər/, e.g. verklaren /vər'k larə/ 'to declare'). The prefix 'ver-' is a verbalizing prefix expressing a change, and the prefix 'be-' is used to change the direction of an action or making an intransitive verb transitive (Booij, 1999). The prefix 'ge-' is often used to create past participles, but can also occur as a verbal or nominal prefix. We focused on verbs with the unproductive prefix ge-. Out of the 80 infinitives, 31 infinitives started with ver-, 31 with be-, and 18 with the prefix ge-. We only selected infinitives with stems that start with consonants. Absence of the schwa in the reduced forms resulted in illegal consonant clusters in $83.75 \%$ of the cases.

In addition, we selected 120 filler infinitives in order to make the stimuli better represent the Dutch lexicon, which contains many infinitives not starting with ver-, be- or ge-. Out of these 120 infinitives, 100 did not start with any prefix (e.g. ademen 'to breathe'), 20 started with prefixes other than be-, ge- or ver- (e.g. ontkennen 'to deny') and nine contained the stem of one of the 80 experimental infinitives (e.g. filler schieten 'to shoot' vs. experimental item beschieten).

A male native speaker of Dutch recorded the infinitives in sentences at sentence-final position (see Experiment 2). From these recordings, we spliced the full and reduced infinitives, using Praat (Boersma \& Weenink, 2014). In all full forms, the prefixal schwa was present, with a mean duration of $43 \mathrm{~ms}$, while in all reduced forms the prefixal schwa was absent. The mean durations of the full and reduced forms were $782 \mathrm{~ms}$ and $739 \mathrm{~ms}$, respectively.
The total item set list contained 200 infinitives. We created two lists; each with all fillers and half of the experimental infinitives in their full forms and the other half in their reduced forms. An infinitive that was reduced in one list, was produced in full in the other list, and vice versa. We pseudo-randomized these lists, ensuring that no more than three experimental infinitives or two infinitives with the same prefix followed each other. The lists were then divided in three blocks, and we ensured that each block started with at least one filler infinitive. Every list was presented to one participant.

\subsubsection{Procedure}

Participants were tested in a dimly-lit soundproof booth, seated in front of a computer, and asked to attentively listen to the infinitives. These infinitives were aurally presented in a short practice session of five trials and three blocks of approximately $5 \mathrm{~min}$, resulting in $17 \mathrm{~min}$ for the whole experiment (Presentation software, Neurobehavioral Systems, www.neurobs.com). A trial started with a fixation cross of $1000 \mathrm{~ms}$, followed by a $300 \mathrm{~ms}$ blank screen, after which the auditory stimulus was played. The next trial started after $3000 \mathrm{~ms}$.

After each block, we presented the participants with a short item-recognition task to ensure that they attentively listened to the stimuli. Participants saw pairs of infinitives on the screen, and were asked to indicate which of two infinitives they had heard in the previous block (e.g. kloppen 'to knock' / klappen 'to clap'). After the task, participants could take a short break.

\subsubsection{EEG recordings}

EEG data were collected from $26 \mathrm{Ag}-\mathrm{Ag} \mathrm{CI}$ electrodes positioned according to the 10-20 standard system. Bipolar horizontal and vertical electrooculograms (EOG) were recorded for ocular artifact rejection. The left mastoid served as the reference electrode and an additional electrode was placed on participants' right mastoid for re-referencing offline. Electrode impedances were kept below $5 \mathrm{k} \Omega$. The EEG was recorded continuously with a band-pass filter of $0.02-100 \mathrm{~Hz}$ and digitized with a sampling frequency of $500 \mathrm{~Hz}$.

\subsubsection{EEG data analysis}

We used FieldTrip (Oostenveld, Fries, Maris, \& Schoffelen, 2011), to pre-process the raw EEG data. We first re-referenced the EEG data offline to the average of the left and right mastoids, and filtered the data with a $0.3-100 \mathrm{~Hz}$ band-pass filter. We then segmented the data into 80 epochs from $-0.5 \mathrm{~s}$ to $2.5 \mathrm{~s}$ relative to the onset of the infinitives. We applied Independent Component Analysis (ICA using the 'runica' implementation) and removed ocular ICA artifacts by visual inspection. On average, we removed 2.32 $(\mathrm{SD}=0.60)$ components per participant in Experiment 1, 2.13 $(\mathrm{SD}=0.57)$ in Experiment 2 and $2.33(\mathrm{SD}=0.73)$ in Experiment 3. After ICA, single trials that still contained artifacts were removed by a semi-automatic rejection routine. On average, 76 out of 80 trials were left for each participant per experiment (mean full forms: Experiment 1: 37.82, SD: 2.02; Experiment 2: 38.06, SD: 1.92; Experiment 3: 37.81, SD: 2.09, mean reduced forms: Experiment 1: 38.08, SD: 1.81; Experiment 2: 37.56, SD: 2.20; Experiment 3: 37.63, SD: 2.40$)$.

\subsubsection{Time-frequency analysis}

We carried out the time-frequency analysis in FieldTrip. Timefrequency (TF) representations of the single-trial data were computed in two frequency ranges. In the first, low frequency range (4-22 Hz), a single taper approach with a 500-ms Hanning window was used to compute power changes in frequency steps of $1 \mathrm{~Hz}$ and time steps of $10 \mathrm{~ms}$. In the high frequency range $(30-80 \mathrm{~Hz}), \mathrm{a}$ multitaper approach (Mitra \& Pesaran, 1999) was used for computing the time-frequency representations, with a 400-ms 
time-smoothing and a $5-\mathrm{Hz}$ frequency-smoothing window, in steps of $1 \mathrm{~Hz}$ and $10 \mathrm{~ms}$. We separately averaged the power estimates for the trials with full and reduced stimuli and used a baseline correction on the time-frequency decomposition of the data from $-500 \mathrm{~s}$ to $0 \mathrm{~ms}$ relative to infinitive onset.

Cluster-based random permutation tests (Maris \& Oostenveld, 2007) were used to evaluate the difference in time-frequency responses per condition and to test the difference between conditions. We used a multi-level statistical approach: On the first level, a dependent-samples-t-test was performed for every data point of two conditions (time by frequency by electrode). All adjacent datapoints that exceeded a pre-set threshold of 5\% (two-tailed) were grouped into clusters. In each of these clusters, the $t$-statistics were summed in order to calculate the cluster-level statistics. Then at the second level, a Monte-Carlo permutation distribution was created by randomly assigning a participant's average to one of the two conditions (1000 times) and calculating the largest clusterlevel statistic for every permutation. The highest cluster-level statistic from each randomization was entered into the MonteCarlo permutation distribution and cluster-level statistics were calculated for the measured data and compared against this permutation distribution. Clusters that fell in the highest or lowest 2.5th percentile of the distribution were considered significant (see Maris and Oostenveld (2007).

\subsection{Results}

\subsubsection{Behavioral results: item-recognition task}

Participants made on average 1.30 mistakes in the recognition task after the first block ( $S D=0.91)$ and 1.13 mistakes in the recognition task after the second block $(S D=0.93)$. This difference between the two blocks was not significant $(t(29)=0.76, p>0.1)$.

\subsubsection{Time-frequency data}

The top row of Fig. 1 displays the grand-average TFRs for the 4-22 Hz frequency range, split for the full and reduced infinitives. A cluster-based permutation test revealed a significant difference between the full and reduced infinitives in the alpha frequency range $(8-12 \mathrm{~Hz})$ : the analysis revealed two positive clusters (one between 1.50 and $1.82 \mathrm{~s}, p<0.05$ and one between 2.05 and $2.50 \mathrm{~s}, p<0.05$ ). These clusters showed the largest differences over posterior and central electrodes, namely Pz, P3, P4 and Cz.

Fig. 2 shows the grand average TFRs for the high frequency range $(30-80 \mathrm{~Hz})$ for the full and reduced stimuli. In response to the full infinitives, we found a small relative increase of high gamma power $(70-80 \mathrm{~Hz})$ and mid-gamma power $(40-60 \mathrm{~Hz})$. The reduced infinitives yielded an opposite effect: a relative decrease of power in the mid-gamma frequency range (40$55 \mathrm{~Hz}$ ). Comparison of the two types of patterns revealed a significant difference between the two stimulus types: our analysis revealed one significant cluster $(0.5-1.7 \mathrm{~s}, p<0.05)$ in the midgamma frequency range, which was most pronounced between 45 and $50 \mathrm{~Hz}$, and over central and posterior electrodes $(\mathrm{Pz}, \mathrm{Cz}$, P3, P4, Cp6, Cp4). Post hoc analyses revealed a low negative correlation between the clusters identified as alpha increase clusters and gamma increase clusters for the full forms $(r=0.26, p=0.07)$ and a strong negative correlation between increased alpha power and decreased gamma power in for reduced forms $(r=-0.75$, $p<0.001)$. Fig. 3 shows that nearly all participants show a similar direction of change in alpha and gamma power in response to the full and reduced stimuli. Please see our Supplementary materials (Figs. S1-S4) for individual alpha and gamma power modulation plots per condition.

To test for possible confounding effects of fatigue or learning, we compared activity between the first and second half of the experiment. We found this difference neither for the low-frequency range nor for the high-frequency range (all $p s>0.1$ ) (note that the absence of a difference does not exclude these possibilities).

In summary, Experiment 1 showed that reduced infinitives elicited larger alpha power increases than full infinitives, while full but not reduced infinitives elicited gamma power increases. In Experiment 2 we investigated whether these differences between full and reduced infinitives also emerge if the reduced infinitives occur in sentence-final position, where they are more frequent than in isolation.

\section{Experiment 2: words in sentence-final position}

\subsection{Methods}

\subsubsection{Participants}

We tested thirty-six right-handed participants (mean age $=21.7, S D=1.8$ ). Six participants were excluded from further analysis because of excessive artifacts in the EEG (2) recording or technical failures (4).

\subsubsection{Materials}

We created 200 sentences consisting of 8 syllables followed by the word forms that were used in Experiment 1 (e.g., Ik zal de sleutel in de kast verstoppen, 'I will the key in the closet hide') All word forms functioned as infinitives and were preceded by auxiliary verbs. Sentence accent was never on these word forms or on the directly preceding syllables.

Seven native speakers of Dutch read the 80 target sentences, which were presented without these target word forms. We asked these participants to complete these sentences by writing down the first three words that came to mind. In $87.9 \%$ of the participants' responses to the sentences, the target verb form was not mentioned at all. In $1.73 \%$ of the responses, the participant mentioned the target verb form as a first choice. In the remaining responses, the target word form was only mentioned as second or third choice. Importantly, there was no verb form that was correctly guessed by more than two participants. This shows that the target infinitives were hardly predictable given the preceding sentential context.

A male native speaker of Dutch recorded the sentences in a soundproof booth, at a sampling rate of $44.1 \mathrm{kHz}$, three times: once without having received any instructions, once with the instruction to pronounce the infinitive in full, once with the instruction to pronounce the infinitive without the prefixal schwa. In the sentences that the speaker produced without having received any instructions, prefixal schwa was present in 52.5\% of the infinitives and absent or unclear in the remaining $47.5 \%$ of the infinitives.

We applied cross-splicing, that is, we replaced the full and reduced forms of the infinitives that the speaker produced without having received any instructions by the full and reduced forms that he produced when explicitly asked to produce these forms. This way, all carrier sentences were identical in the reduced and full condition. This way, all carrier sentences were identical in the reduced and full condition. We used the same experimental lists as in Experiment 1.

\subsubsection{Procedure}

The procedure was identical to the procedure in Experiment 1 and lasted $25 \mathrm{~min}$ in total. In a recognition task after each block, participants were asked to indicate which of two sentences they heard in the previous block (e.g. Je zult op die manier je hand verwonden 'You will hurt your hand that way' / Je zult op die manier je arm verwonden 'You will hurt your arm that way'). In most sentences, the direct object of the sentence was altered (as in the example above). 


\section{Experiment 1: Full and reduced words in Isolation}
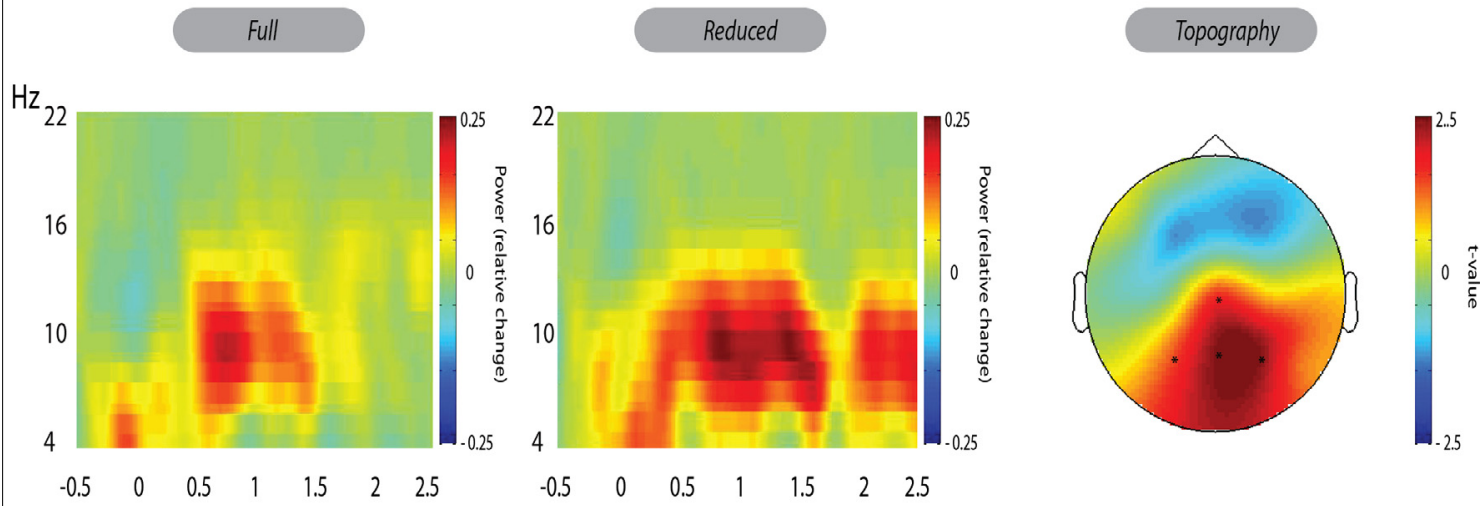

Experiment 2: Full and reduced words in Sentence-final position
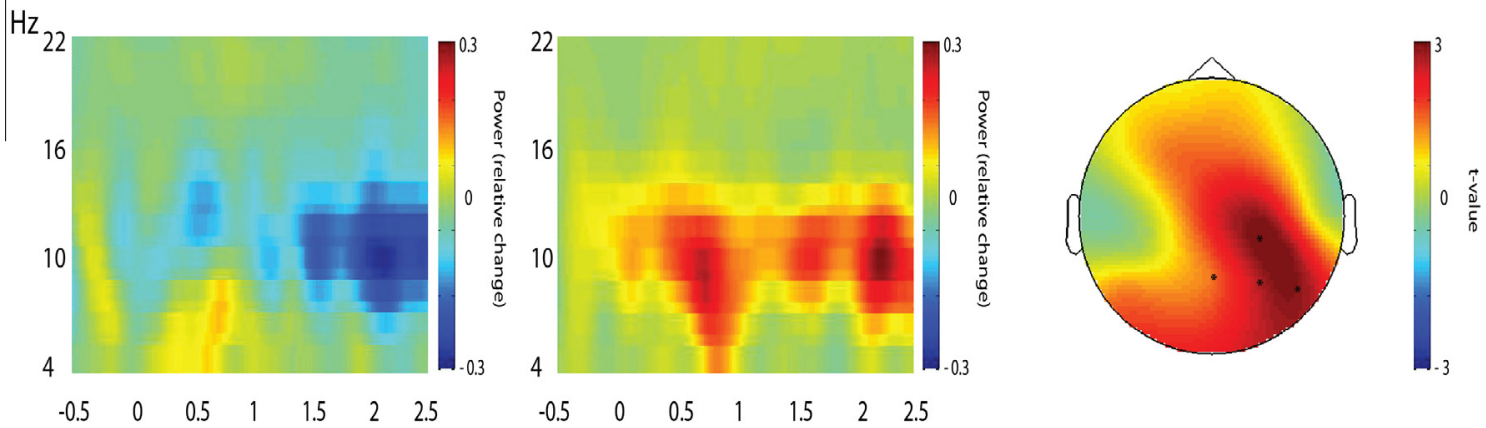

Experiment 3: Full and reduced words in Mid-sentence position
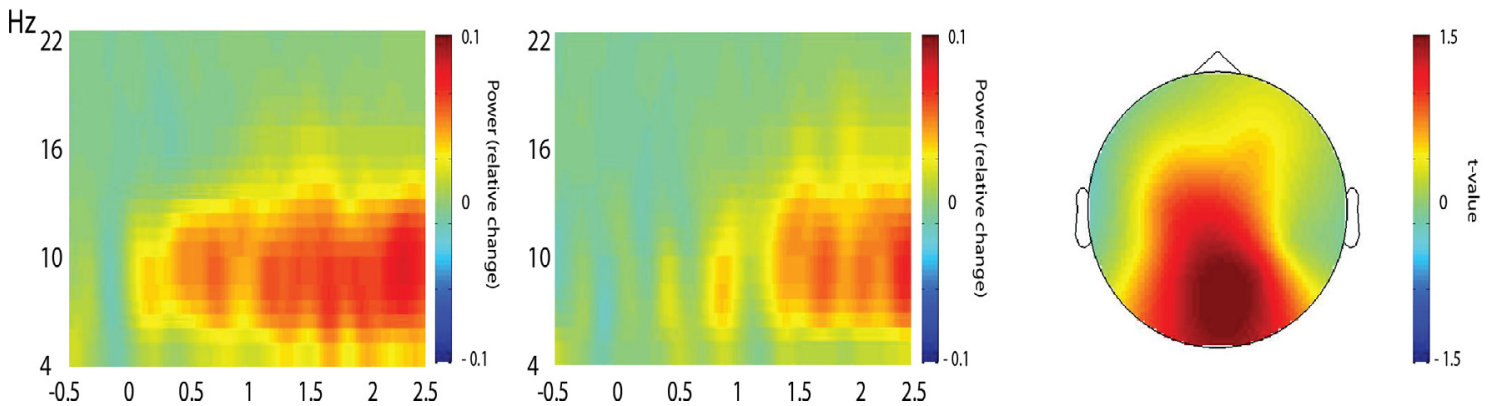

Fig. 1. Results of TF Analysis over significant electrodes per experiment. The left panels show the grand-average TF representations for the low-frequency range for the full conditions and the middle panels show the reduced conditions, all relative to a $500 \mathrm{~ms}$ baseline. The right panels present difference topography plots.

\subsubsection{EEG recordings}

All procedures in EEG recording, Time-Frequency Analysis and Statistical Analyses were identical to those in Experiment 1.

\subsection{Results}

\subsubsection{Behavioral results: sentence-recognition task}

Participants made 0.73 mistakes in the recognition task after block $1(\mathrm{SD}=0.84), 1.00$ mistake after block $2(\mathrm{SD}=0.90)$ and 0.75 mistakes after block $3(\mathrm{SD}=0.76)$. This difference between the blocks was not statistically significant $(F(1.915,61.279)$ $=1.044, p>0.1$ ).

\subsubsection{Time-frequency data}

The middle row of Fig. 1 displays the grand average TFRs for the sentences with the full and reduced infinitives in the alpha frequency range $(8-12 \mathrm{~Hz})$. The TFR of the full infinitives shows a relative decrease of alpha power between $1.7 \mathrm{~s}$ and $2.5 \mathrm{~s}$ after word onset. The TFR of the reduced infinitives displays a relative increase in alpha power between 0.3 and $2.5 \mathrm{~s}$ after word onset. Interestingly, the enhancement for the reduced infinitives started about $400 \mathrm{~ms}$ before the offset of the target word. This suggests that alpha power increases as soon as an illegal consonant cluster is encountered and an expected vowel (schwa) is absent.

A cluster-based permutation test revealed a significant difference between the two conditions and identified two positive clusters in the alpha frequency range between $0.5 \mathrm{~s}$ and $0.9 \mathrm{~s}(p<0.05)$ and between 1.7 and $2.50 \mathrm{~s}(p<0.01)$ measured from word onset (full $<$ reduced in both clusters) over posterior electrodes (Pz, P4, P3, P7). Post hoc comparisons of the differences in alpha power between full and reduced forms in the first and second part of Experiment 2 or between Experiment 1 vs. Experiment 2 revealed no significant interactions ( $p s>0.1$ ).

The middle row of Fig. 2 shows the grand-average TFRs in the gamma frequency range for the full and reduced forms. In the high frequency range $(30-80 \mathrm{~Hz})$, the cluster-based permutation test 


\section{Experiment 1: Full and reduced words in Isolation}
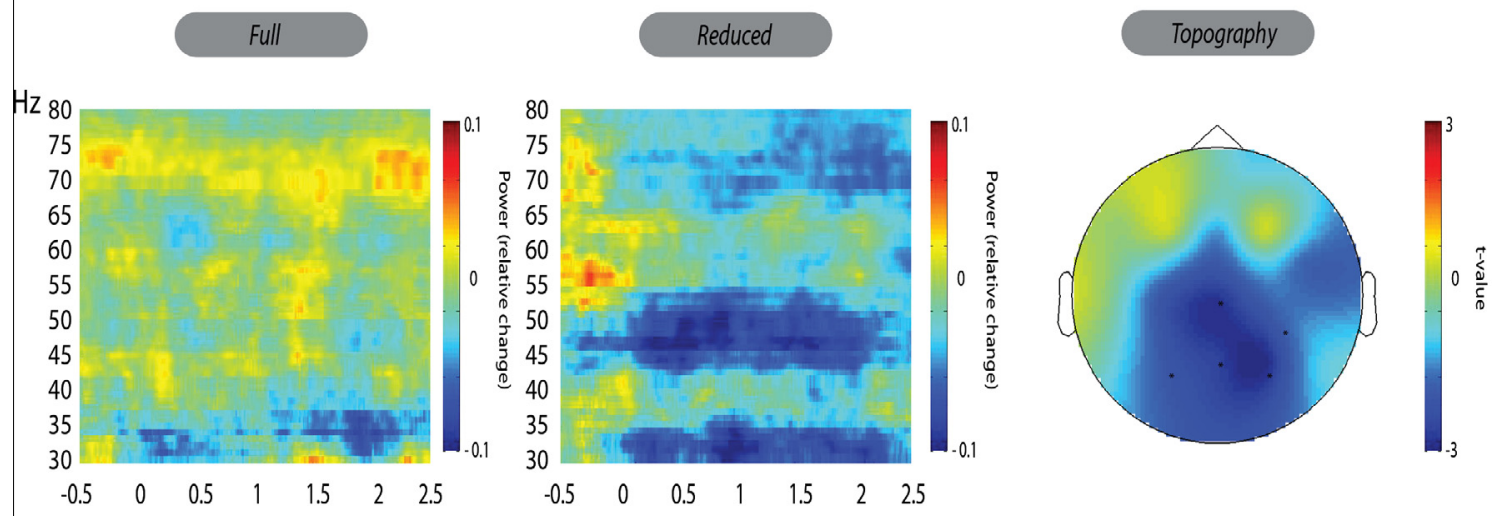

Experiment 2: Full and reduced words in Sentence-final position
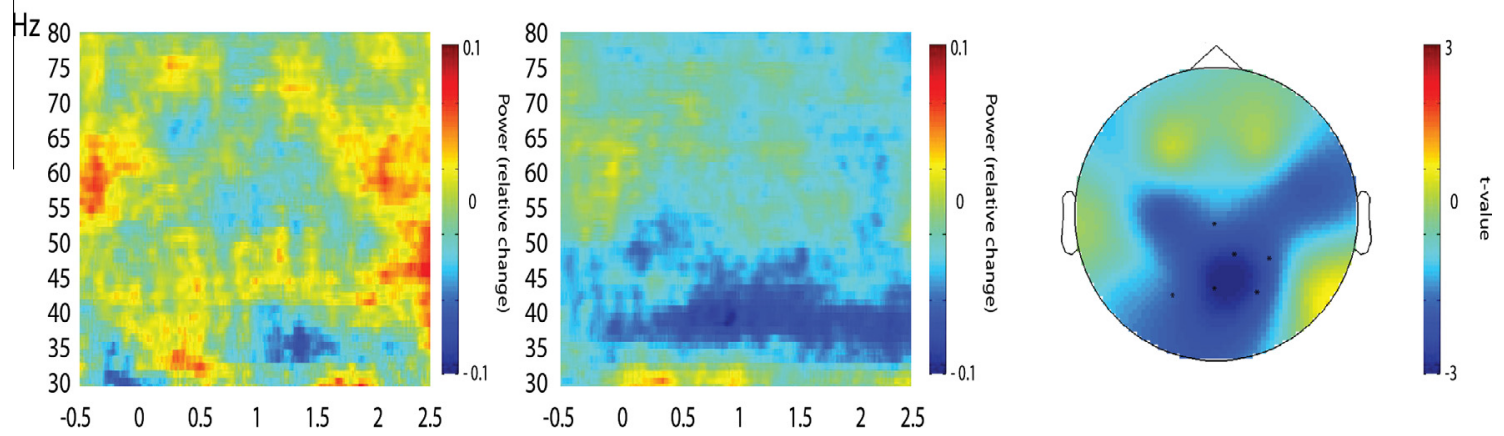

Experiment 3: Full and reduced words in Mid-sentence position
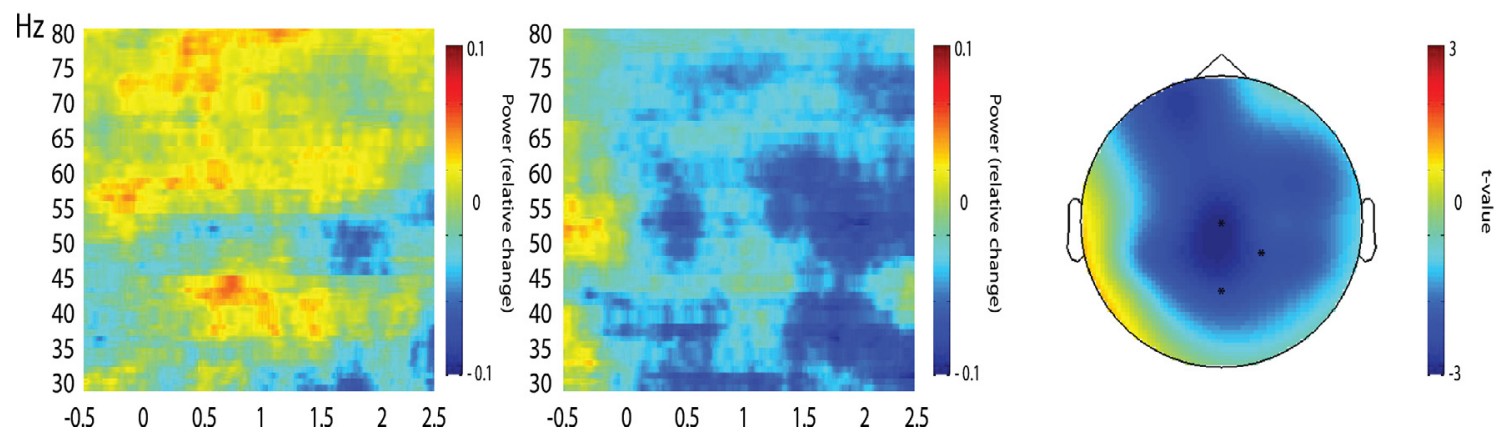

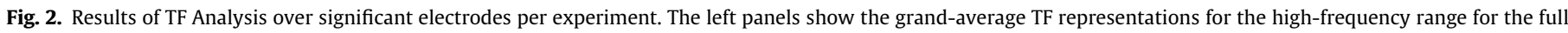
conditions and the middle panels show the reduced conditions, all relative to a $500 \mathrm{~ms}$ baseline. The right panels present difference topography plots.

revealed a significant difference $(p<0.01)$ between the full and reduced infinitives (one positive cluster between approximately 37 and $46 \mathrm{~Hz}$, from $0.5 \mathrm{~s}$ to $1.4 \mathrm{~s}$, over central and posterior electrodes Pz, P3, C3, Cz and CP5). A post hoc analysis of the relation between alpha and gamma power revealed a moderate negative correlation for both the full $(r=-0.54, p<0.001)$ and reduced forms $(r=-0.48, p<0.001)$. A post hoc pairwise comparison of the difference in gamma power between full and reduced forms in Experiment 1 vs. Experiment 2 revealed no significant interaction $(p>0.1)$. A comparison between the relative power changes in the first and second half of the experiment revealed no significant differences either (full and reduced $p>0.1$ ). Fig. 3 shows that nearly all participants show a similar direction of change in alpha and gamma power in response to the full and reduced stimuli. Please see Figs. S5-S8 for individual alpha and gamma power modulation plots per condition.

Experiment 2 investigated whether oscillatory power modulations in the alpha and gamma frequency band could inform us on how the processing of full and reduced words differs in sentence-final position. As in Experiment 1, we found larger alpha power increases in response to reduced words in than in response to full words, while gamma power only increased in response to full words, but not in response to reduced words. In response to full words, we observed an alpha suppression.

Schwa-reduction occurs most frequently in words in the middle of an utterance. Therefore, reduced word processing in midsentence position may be easier for listeners than in sentencefinal position or in isolation. This was investigated in Experiment 3.

\section{Experiments 3: words in mid-sentence position}

\subsection{Methods}

\subsubsection{Participants}

We tested thirty right-handed participants (mean age $=20.9$, $\mathrm{SD}=2.1$ ). Three participants were excluded from further analysis 

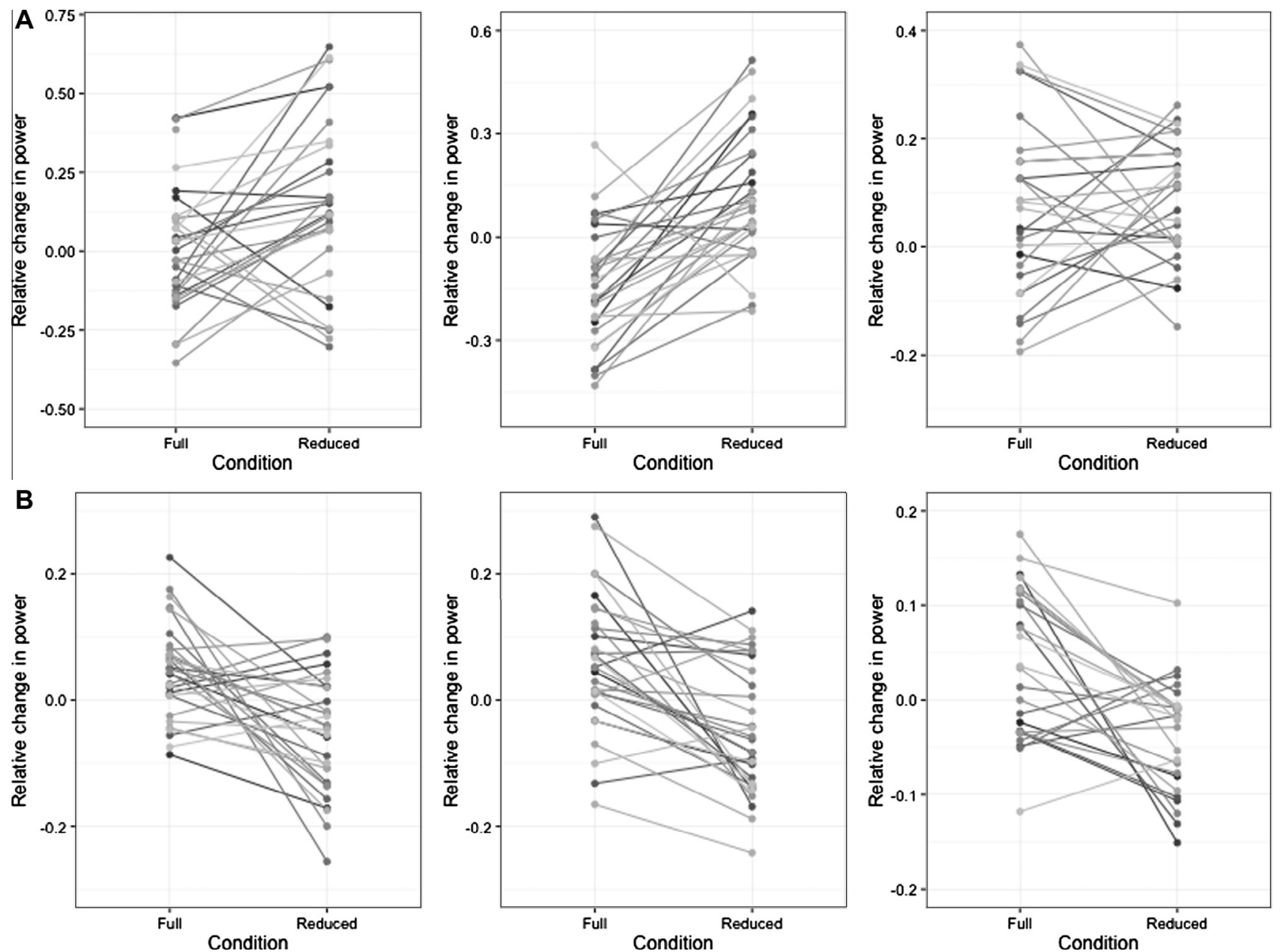

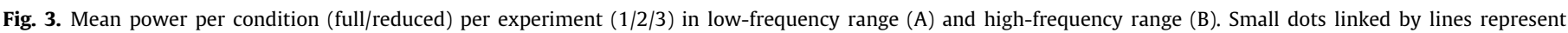
individual participants.

because of excessive artifacts in the EEG recording (2) or technical failures (1).

\subsubsection{Stimulus materials}

The same experimental verb forms were used as in Experiments 1 and 2. New sentences were recorded by the same male native speaker of Dutch who recorded the stimuli for the previous experiments. In these new sentences, the verb forms occurred in mid-sentence position (e.g., De kinderen verstoppen zich achter de schutting van de tuin, 'The children are hiding behind the fence of the garden'.). We used the same filler sentences as in Experiment 2.

In mid-sentence position, the verb forms could serve multiple syntactic functions. The verb was the main verb of the predicate in 30 sentences (e.g., De bewoners bereiden de open dag voor, 'The inhabitants prepare the open day'); it was preceded by an auxiliary verb in 26 sentences (e.g., Hij wil alles verdelen over zijn kleinkinderen 'He wants everything to divide over his grandchildren',) and it was part of the subject in 24 sentences (e.g., Het jongetje genezen was voor deze arts niet moeilijk, 'The boy curing was for this doctor not hard'). By having the verb occur with different syntactic functions, we ensure that our results do not depend on a specific syntactic construction.

The target verb form was always preceded by four syllables. Sentence accent was never on the target verb form or the syllable preceding this verb form. To control for predictability effects, the semantic context up until the target verb form was kept as neutral as possible.
Eight native speakers of Dutch were visually presented with the 80 sentences up to the target word forms and were asked to complete these sentences by writing down three words that came to mind. In $97.8 \%$ of the participants' responses, none of the target verb forms were mentioned at all. The target verb form was mentioned as the first choice in $1.39 \%$ of the responses, as the second choice in $0.31 \%$ and as the third choice in $0.46 \%$ of the responses. This shows that the target infinitives were not highly predictable given the preceding sentential context.

We reused the recordings of the filler sentences from Experiment 2. The experimental sentences were recorded as in Experiment 2 . That is, we recorded them three times: once without the speaker having received any instructions, once with the instruction to pronounce all verb forms in full, once with the instruction to pronounce the verb forms without the prefixal schwas. In the sentences that the speaker produced without having received any instructions, prefixal schwa was present in $68.4 \%$ of the verb forms and absent or unclear in the $31.6 \%$ of the verb forms. We applied the same cross-splicing method as in Experiment 2 to ensure that all sentences were identical in the reduced and full condition, except for the realization of the target verb form in the middle of the sentence. In all full forms, the prefixal schwa was present and had a mean duration of $42.24 \mathrm{~ms}$, while in all reduced forms the prefixal schwa was absent. The mean durations of the full and reduced forms were $495 \mathrm{~ms}$ and $430 \mathrm{~ms}$, respectively.

The experimental lists were constructed in the same manner as in Experiment 1 and Experiment 2. 
4.1.3. Procedure

The design and procedure were identical to those in Experiment 2.

\subsubsection{EEG recordings}

To avoid any confounding effects due to mid-sentence oscillatory activity, we used a $500 \mathrm{~ms}$ pre-sentence baseline, instead of a baseline that directly preceded the experimental infinitives. All remaining procedures in EEG recording, time-frequency analysis and statistical analyses were identical to those in Experiment 1 and Experiment 2.

\subsection{Results}

\subsubsection{Behavioral results: sentence-recognition task}

The performance on the sentence-recognition task revealed that on average, participants made 0.43 mistakes after block 1 $(\mathrm{SD}=0.68), 0.30$ mistakes after block $2(\mathrm{SD}=0.46)$ and 0.43 mistakes after block $3(\mathrm{SD}=0.62)$. This difference between blocks was not statistically significant $(F(1.812,52.560)=0.049, p>0.1)$.

\subsubsection{Time-frequency data}

Fig. 1 (lower row) shows the grand-average TFRs of the full and reduced stimuli. A general analysis of the low frequency range (4$22 \mathrm{~Hz})$ yielded no significant differences $(p>0.1)$, neither did a focused analysis of the alpha band $(8-12 \mathrm{~Hz}$, averaged over frequencies; $p>0.1$ ). A comparison of the relative power changes in the alpha band between the first and second half of the experiment revealed no significant effects for neither the full $(p>0.1)$ nor the reduced stimuli $(p>0.1)$. Additionally, there were no differences in alpha power between the three different syntactic functions within and between the full and reduced stimuli $(p>0.1)$.

The lower row of Fig. 2 displays the grand average TFRs of the full and reduced stimuli and their topographical distribution in the high-frequency range $(30-80 \mathrm{~Hz})$. A general cluster-based permutation test over the whole frequency range $(30-80 \mathrm{~Hz})$ yielded a single positive cluster in the mid-gamma frequency range (approx. $40-45 \mathrm{~Hz}$ ) from 0.6 to $1.0 \mathrm{~s}$, occurring over central-parietal electrodes $\mathrm{Cz}, \mathrm{Pz}$ and $\mathrm{CP} 3$, indicating a significant difference between the two stimulus types $(p<0.05)$. Post hoc analyses revealed no correlation between alpha and gamma power for sentences with full forms $(r=0.15, p=0.15)$ and a strong correlation between alpha and gamma power for sentences with reduced verb forms $(r=-0.65, p<0.001)$. Additionally, post hoc pairwise comparisons revealed significant differences in the difference in alpha power between full forms and reduced forms for Experiment 1 versus Experiment $3(p<0.05)$ and for Experiment 2 versus Experiment $3(p<0.05)$, but not in the difference of gamma power (all $p s>0.1$ ). A comparison between the oscillatory responses in the first and second half of the experiment revealed no significant differences $p$ s for full and reduced $>0.1$ ). Additionally, there were no differences in gamma power between the three different syntactic functions in the full and reduced stimuli $(p>0.1)$. Please see Figs. S9-S12 for individual alpha and gamma power modulation plots per condition.

\section{General discussion}

This study investigated oscillatory power modulations in the alpha and gamma frequency bands in order to gain more insight into how listeners process reduced versus full word forms and how this is modulated by linguistic context. Importantly, studying oscillatory activity can provide a more fine-grained account of how reduced and full forms are processed than behavioral paradigms because it can inform about how words are processed within larger speech units (e.g. sentences) and how the brain allocates resources to optimize reduced and full form processing.

Behavioral studies have reported a processing advantage for full forms compared to reduced forms when these forms are presented in isolation (e.g., Ernestus et al., 2002; Janse \& Ernestus, 2011; Tucker \& Warner, 2007) or at the end of simple sentences (e.g. Bürki et al., 2010; Ranbom \& Connine, 2007). However, given the finding that reduced forms occur most frequently in the middle of full sentences (e.g., Pluymaekers et al., 2005) and that full forms lose some of their special status when they are presented in context (e.g., Bürki et al., 2010), the processing advantage for full forms was expected to disappear in connected speech. In this study, we studied oscillatory power in the alpha and gamma frequency bands in response to reduced and full forms in different speech contexts: In a passive-listening task, Dutch native listeners were presented with Dutch full and reduced verb forms in isolation (Experiment 1 ), in sentence-final position (Experiment 2) and in mid-sentence position (Experiment 3).

We observed larger alpha power in response to reduced compared to full word forms in isolation (Experiment 1) and in sentence-final position (Experiment 2), but not in mid-sentence position (Experiment 3). Moreover, in all three experiments, we observed a small relative increase in gamma power in response to full forms, but not in response to reduced forms. The observed oscillatory power differences in the alpha and gamma frequency band suggest that reduced forms and full forms are processed differently. Below, we discuss what these modulations of alpha and gamma power may reveal about the processing of reduced and full forms in the three different linguistic contexts.

Previous research has suggested that posterior alpha oscillations reflect a mechanism that inhibits task-irrelevant brain areas or distracting neural activity. This inhibitory mechanism has been observed in tasks involving working memory and selective attention in the visual domain, as well as in experiments that tap into language comprehension, such as studies on degraded speech processing (e.g., Jensen \& Mazaheri, 2010; Strauß, Kotz, et al., 2014; Strauß, Wostmann, et al., 2014). Previous research provided evidence that increased alpha power can be a reliable indicator of a higher cognitive load in audition (for a discussion, see Strauß, Wostmann, et al., 2014). For instance, a parametric increase of alpha power was observed in response to increasingly degraded speech signals (Obleser \& Weisz, 2012), in experiments in which temporal expectations about the upcoming signal were varied (e.g. the expectancy of the occurrence of a syllable in time; Wilsch, Henry, Herrmann, Maess, \& Obleser, 2014), in which auditory memory load and acoustic degradation were parametrically varied in a Sternberg paradigm (e.g. Obleser et al., 2012, see for a full review Strauß, Kotz, et al., 2014), and when ambiguous pseudowords (i.e., real words with one altered segment) were compared to pseudowords and real words (Strauß, Kotz, et al., 2014; Strauß, Wostmann, et al., 2014).

We observed larger alpha power in response to reduced compared to full forms in isolation and in sentence-final position. Based on the functional inhibition framework, an initial explanation could be that processing reduced forms taxes lexical processing more than full forms do: Listening to reduced forms can be challenging because part of the speech signal is absent or difficult to interpret. This may especially hold for words presented in isolation and in sentence-final position, as reduced forms are less frequent in these positions. As a consequence, the participants' auditory system may have directed more attention to the speech signal. This would result in more processing effort and a higher auditory cognitive load during the processing of reduced forms compared to full forms. This interpretation is in line with findings from several experimental studies using adverse listening conditions (see for a full review Strauß, Kotz, et al., 2014). Here, 
enhanced alpha power is proposed to aid in neurally gating reduced words towards integration with a meaning representation.

In mid-sentence position, we observed a small increase in alpha power in response to reduced forms but this was not statistically different from the increase in alpha power for full forms. We propose that the small increases in alpha power for full and reduced forms could reflect general inhibitory processes that were active during the processing of information that followed the target word. This was not the case in the first two experiments because no additional information needed to be processed after the target words.

In contrast, we observed post-stimulus alpha suppression in response to full forms in sentence-final position. Previous research has suggested that post-stimulus alpha suppression reflects a state that allows for an increase of mental operations that can be performed on the incoming speech signal, thus, active cognitive processing (e.g., Obleser \& Weisz, 2012; Obleser et al., 2012; Shahin et al., 2009; Strauß, Kotz, et al., 2014; Strauß, Wostmann, et al., 2014). In line with these studies, we suggest that the observed alpha suppression reflects more effective language processing, allowing for more mental operations that can be performed on the speech signal, with alpha oscillations in response to full forms being suppressed as a function of auditory cognitive load in processing.

We did not find an alpha suppression in response to full forms in mid-sentence position or isolation. A possible explanation for the absence of this suppression in isolation is that both full and reduced forms may have caused more processing effort and auditory cognitive load because they were presented outside of a sentence context. Although both types of forms elicited enhanced alpha power, we observed larger alpha power in response to reduced words compared to full forms. This indicates that reduced words imposed a higher auditory cognitive load on processing than full forms.

Moreover, we did not observe alpha suppression in response to full forms in mid-sentence position. A possible explanation is that the verb forms in mid-sentence position did not carry accent and were relatively short as they were not located at the edges of strong prosodic boundaries (unlike the sentence-final words or the words presented in isolation). Therefore, there was probably less attentional focus on the actual form of the verb forms, and reduced forms did not elicit more processing effort than full forms. Alternatively, the processing advantage for full forms might disappear in mid-sentence position because reduced forms tend to occur more often in mid-sentence position (Pluymaekers et al., 2005).

Although our experiments did not involve any visual input, we considered the possibility that non-auditory generators of alpha (such as the visual system) may have modulated the results we found on alpha activity. We observed alpha power increases over (central)-parietal electrodes in the first two experiments, which suggests that (central)-parietal areas were inhibited to selectively gate the information that came from our auditory stimuli, allowing for active engagement of language and auditory-related areas. Importantly, we did not find significant differences in alpha power increases over occipital areas. This suggests that the observed alpha power changes were not a result of non-relevant visual activity.

Moreover, the difference topographies from the first two experiments revealed a left frontotemporal alpha suppression. This differential modulation forms a strong suggestion for different processes in auditory and visual areas and supports the existence of an auditory alpha mechanism (see for a discussion: Strauß, Wöstmann, et al., 2014; Weisz, Hartmann, Müller, Lorenz, \& Obleser, 2011). In addition, alpha modulations over more domain-general areas such as the superior parietal cortex have shown to be indicative of successful speech comprehension (Obleser \& Weisz, 2012; Weisz et al., 2011). Therefore, we do not interpret the results we found as an epiphenomenon of visual activity. Future research could address this issue further by including source reconstruction in the analyses. Moreover, future research that uses MEG could tap into the question if a possible auditory alpha mechanism can be reliably separated from visual alpha.

As for gamma activity, in all three experiments, we observed a small increase of gamma power in response to full forms, but not in response to reduced forms. These gamma increases were present at approximately $40-45 \mathrm{~Hz}$ over central-parietal electrodes. So far, gamma power increases in language comprehension research have mostly been associated with semantic unification processes (see e.g. Bastiaansen et al., 2012) such as assessing the predictability of an upcoming word based on the preceding context (Wang et al., 2012), the activation of local functional networks that support semantic representations (Mellem et al., 2013), and the successful matching of top-down lexical memory traces upon understanding degraded auditory stimuli (Hannemann et al., 2007). More generally, gamma power increases are thought to reflect active, neuronal processing, mediating feature binding (e.g. Jokisch \& Jensen, 2007). Moreover, it has been proposed that increases in gamma power should be observed when bottom-up information, such as stimulus-related information (e.g. an incoming word), matches the contents of top-down pre-activated memory information (Herrmann, Munk, \& Engel, 2004). Additionally, increased gamma power during sentence processing has been hypothesized to reflect the facilitated integration of high-cloze sentence final words into sentential meaning (Obleser et al., 2011). In line with this, a recent account on the role of gamma activity in language comprehension proposes that gamma activity during language comprehension reflects an index of how well the actual (speech) input matches a lexical item (in long-term memory) that has been pre-activated as a prediction of the upcoming linguistic input. These predictions are based on the preceding context, in combination with other information that the language processing system considers relevant in making this prediction (e.g. sentence/discourse information) (Lewis \& Bastiaansen, 2015; Lewis, Wang, \& Bastiaansen, 2015).

In all three experiments, full forms, but not reduced forms elicited gamma power increases (relative to baseline). The reduced forms were comprehendible and intelligible (as indexed by the scores on the recognition task in all experiments). It is therefore unlikely that the gamma increases for full forms uniquely reflect lexical access or retrieval of semantic information, since this should be the case for reduced forms as well.

Additionally, it is also unlikely that the observed gamma increases in response to full forms were caused by the predictability of the occurrence of our stimuli. The results from the rating tasks indicate that the verb forms were not highly predictable. The word class was probably predictable to some extent (i.e., a verb), but not the stem of the verb form or the presence of a prefix (there were a large number of fillers that did not contain prefixes) nor the occurrence of a full or reduced form. Therefore, we believe that the predictability of our stimuli cannot explain the increases in gamma power that were found in all three experiments in response to the full forms.

Our tentative explanation for the observed gamma power increases in response to full forms is that these increases reflect spreading of activation through networks containing semantic representations. The absence of gamma power increases in response to reduced words suggests that processing reduced variants requires more processing time and processing effort before activation can fully spread through semantic networks to activate the corresponding meaning representation. This explanation extends behavioral results of studies on acoustic reduction which suggest that, after hearing a reduced prime, listeners need more time to 
process the subsequent semantically related target word if the prime word is reduced than if it is full and that reduced forms require a processing cost to be recognized (e.g. Tucker \& Warner, 2007; Van de Ven et al., 2011).

In line with the behavioral results of Van de Ven et al. (2011), we propose that when reduced forms impose a higher auditory cognitive load on lexical processing (as indexed by enhanced alpha power), this can impede or delay activation spreading through semantic networks (as indexed by a decrease in gamma power relative to baseline). The higher auditory cognitive load associated with processing reduced forms could result in a delayed activation of the corresponding meaning representation. This processing cost might occur because the higher auditory cognitive load during reduced form processing needs to be resolved before the corresponding meaning representation can be activated. Similar results have been found in behavioral studies that investigated attentional and perceptual factors in lexical processing and semantic priming. For instance, Smith, Bentin, and Spalek (2001) argued that activation-spreading in a semantic network does not occur while increased cognitive load is not yet resolved (the attention modulation hypothesis). Other studies on semantic priming have substantiated this hypothesis by showing that semantic priming is modulated by the amount of attentional resources (Otsuka \& Kawaguchi, 2007), semantic activation does not fully spread to semantic associates when speech is degraded or when a prime word is not salient enough in a dichotic listening situation (Aydelott \& Bates, 2004; Dupoux, Kouider, \& Mehler, 2003). In line with this, our data suggests that in the case of reduced forms, activation cannot immediately fully spread to a semantic representation, due to the higher auditory cognitive load that reduced forms impose on lexical processing. The fact that gamma increases are absent during the processing of reduced stimuli (and the data even show decreased gamma activity relative to baseline) suggests that the language system needs to resolve this higher auditory cognitive load before the corresponding item can be accessed, which suggests that there might be a delayed or weaker activation of the corresponding meaning representation. Although we did not find a significant difference in alpha power in mid-sentence position, we still observed a difference in gamma power. This suggests that although reduced forms did not impose a higher auditory cognitive load on processing than full forms, full forms still benefit from a processing advantage. Additionally, our post hoc analyses revealed strong correlations between relative suppression of gamma power and a relative increase in alpha power, in all three experiments. This is in accordance with the hypothesis that higher alpha power is associated with functional inhibition of high frequency oscillations, which are thought to reflect active, neural communication and computations (Fries, 2009; Jensen \& Mazaheri, 2010; Osipova, Hermes, \& Jensen, 2008).

In summary, our study extends behavioral research by investigating the processing of reduced and full forms in isolation and in sentential context. Although our manipulation consisted of a subtle difference in the realization of a word, that is, the presence or absence of prefixal schwa, the auditory system was sensitive to these different realizations during passive listening, even when they occurred in connected speech. Schwa reduction modulated oscillatory power in the alpha frequency band for words presented in isolation and in sentence-final position: larger alpha power was observed for reduced forms than for full forms. We argue that this larger alpha power reflects a higher auditory cognitive load, which implies more effortful processing of reduced forms before they are coupled to a meaning representation. As such, alpha oscillations serve as an inhibitory mechanism to gate reduced words towards integration with a meaning representation. This increase in cognitive load for reduced forms was absent in mid-sentence position because reduced forms are more common in this position and the context requires less attentional demands towards the word. In contrast, the relatively lower alpha power, or even alpha suppression, for full words is likely to reflect active and effortless language processing (Obleser \& Weisz, 2012; Strauß, Kotz, et al., 2014; Strauß, Wostmann, et al., 2014).

Moreover, in all three speech contexts, an increase of gamma power was observed in response to full forms but not in response to reduced forms. The absence of gamma power increases in response to reduced forms suggests that the processing of reduced forms requires more processing time and processing effort, which can delay activation spreading through networks of semantic representations. Future studies have to show whether this interpretation is correct.

In conclusion, our results show that speech reduction affects the listening process differently for different speech contexts. Importantly, oscillatory modulations in the alpha and gamma frequency band may provide information about how listeners process full and reduced word forms that cannot be easily revealed in behavioral studies. In the study of differences in oscillatory power, participants may just listen to the auditory stimuli, and the words under investigation can be presented in context, in different sentential positions, which allows, among others, for the study of the role of sentence position in word processing. Our findings show that the observed alpha increases in response to reduced forms reflect a higher auditory cognitive load in lexical processing. Here, alpha oscillations serve as an inhibitory mechanism to neutrally gate reduced forms towards integration with a semantic representation. Additionally, our data suggest that the observed gamma power increases in response to full forms reflect active, neuronal processing, allowing for effortless activation of a semantic representation. The absence of gamma increases in response to reduced forms illustrates that listeners require more processing time and effort to activate semantic representations. We conclude that oscillatory power differences in the alpha and gamma frequency band index differential processing of the full and reduced forms of words that only differ in the presence of schwa, independently of whether the words are presented in context and independently of their positions in the sentence, but especially so for words in isolation or in sentence-final position.

\section{Conflict of interest}

The authors declare no conflict of interest.

\section{Acknowledgements}

This work was supported by an ERC consolidator grant (284108) to the third author. We thank two anonymous reviewers for their constructive comments, which helped to improve this manuscript. Furthermore, we would like to thank Ole Jensen for his comments on an earlier version of this article.

\section{Appendix A. Supplementary material}

Supplementary data associated with this article can be found, in the online version, at http://dx.doi.org/10.1016/j.bandl.2016.01. 003.

\section{References}

Adrian, E. D. (1944). Brain rhythms. Nature, 153, 360-362.

Aydelott, J., \& Bates, E. (2004). Effects of acoustic distortion and semantic context on lexical access. Language and Cognitive Processes, 19(1), 29-56.

Bastiaansen, M. C. M., Mazaheri, A., \& Jensen, O. (2012). Beyond ERP's: Oscillatory neuronal dynamics. In S. J. Luck \& E. S. Kappenman (Eds.), The Oxford handbook of event-related potential components (pp. 31-50). New York, NY: Oxford University Press. 
Boersma, P., \& Weenink, D. (2014). Praat: Doing phonetics by computer [Computer program]. Version 5.4.04. <http://www.praat.org/> Retrieved 28.12.14.

Booij, G. (1999). The phonology of Dutch. Oxford University Press.

Bürki, A., Ernestus, M., \& Frauenfelder, U. (2010). Is there only one "fenêtre" in the production lexicon? On-line evidence on the nature of phonological representations of pronunciation variants for French schwa words. Journal of Memory and Language, 62(4), 421-437.

Dupoux, E., Kouider, S., \& Mehler, J. (2003). Lexical access without attention? Explorations using dichotic priming. Journal of Experimental Psychology: Human Perception and Performance, 29(1), 172-184.

Ernestus, M. (2000). Voice assimilation and segment reduction in casual Dutch a corpus-based study of the phonology-phonetics interface. Utrecht: LOT.

Ernestus, M., \& Baayen, H. (2007). Paradigmatic effects in auditory word recognition: The case of alternating voice in Dutch. Language and Cognitive Processes, 22(1), 1-24.

Ernestus, M., Baayen, H., \& Schreuder, R. (2002). The recognition of reduced word forms. Brain and Language, 81(1-3), 162-173.

Ernestus, M. (2014). Acoustic reduction and the roles of abstractions and exemplars in speech processing. Lingua, 142, 27-41.

Ernestus, M., \& Warner, N. (2011). An introduction to reduced pronunciation variants. Journal of Phonetics, 39, 253-260.

Foxe, J. J., Ca, G. V. S., \& Ahlfors, S. P. (1998). Parieto-occipital approximately $10 \mathrm{~Hz}$ activity reflects anticipatory state of visual attention mechanisms. Neuroreport, 9(17), 3929-3933.

Foxe, J. J., \& Snyder, A. C. (2011). The role of alpha-band brain oscillations as a sensory suppression mechanism during selective attention. Frontiers in Psychology, 2(July), 154. http://dx.doi.org/10.3389/fpsyg.2011.00154.

Fries, P. (2009). Neuronal gamma-band synchronization as a fundamental process in cortical computation. Annual Review of Neuroscience, 32, 209-224.

Fu, K. M., Foxe, J. J., Murray, M. M., Higgins, B. a., Javitt, D. C., \& Schroeder, C. E. (2001). Attention-dependent suppression of distracter visual input can be cross-modally cued as indexed by anticipatory parieto-occipital alpha-band oscillations. Brain Research, 12(1), 145-152.

Hald, L. A., Bastiaansen, M. C. M., \& Hagoort, P. (2006). EEG theta and gamma responses to semantic violations in online sentence processing. Brain and Language, 96, 90-105.

Hannemann, R., Obleser, J., \& Eulitz, C. (2007). Top-down knowledge supports the retrieval of lexical information from degraded speech. Brain Research, 1153, 134-143.

Herrmann, C. S., Munk, M. H., \& Engel, A. K. (2004). Cognitive functions of gammaband activity: Memory match and utilization. Trends in Cognitive Sciences, 8(8), 347-355.

Hipp, J. F., Engel, A. K., \& Siegel, M. (2011). Oscillatory synchronization in large-scale cortical networks predicts perception. Neuron, 69(2), 387-396.

Janse, E., \& Ernestus, M. (2011). The roles of bottom-up and top-down information in the recognition of reduced speech: Evidence from listeners with normal and impaired hearing. Journal of Phonetics, 39(3), 330-343.

Janse, E., Nooteboom, S., \& Quene, H. (2007). Coping with gradient forms of /t/ deletion and lexical ambiguity in spoken word recognition. Language and Cognitive Processes, 22, 161-200.

Jensen, O., \& Mazaheri, A. (2010). Shaping functional architecture by oscillatory alpha activity: Gating by inhibition. Frontiers in Human Neuroscience, 4, 186.

Johnson, K. (2004). Massive reduction in conversational American English. In K. Yoneyama, \& K. Maekawa (Eds.), Spontaneous speech: Data and analysis (pp. 2954).

Jokisch, D., \& Jensen, O. (2007). Modulation of gamma and alpha activity during a working memory task engaging the dorsal or ventral stream. Journal of Neuroscience, 27(12), 3244-3251.

Klimesch, W., Sauseng, P., \& Hanslmayr, S. (2007). EEG alpha oscillations: The inhibition-timing hypothesis. Brain Research Reviews, 53(1), 63-88.

Lewis, A. G., \& Bastiaansen, M. (2015). A predictive coding framework for rapid neural dynamics during sentence-level language comprehension. Cortex. http:// dx.doi.org/10.1016/j.cortex.2015.02.014.

Lewis, A. G., Wang, L., \& Bastiaansen, M. (2015). Fast oscillatory dynamics during language comprehension: Unification versus maintenance and prediction? Brain and Language, 1-13.

Maris, E., \& Oostenveld, R. (2007). Nonparametric statistical testing of EEG- and MEG-data. Journal of Neuroscience Methods, 164(1), 177-190.
Mazaheri, A., van Schouwenburg, M. R., Dimitrijevic, A., Denys, D., Cools, R., \& Jensen, O. (2014). Region-specific modulations in oscillatory alpha activity serve to facilitate processing in the visual and auditory modalities. Neurolmage, 87, 356-362.

Mellem, M. S., Friedman, R. B., \& Medvedev, A. V. (2013). Gamma- and theta-band synchronization during semantic priming reflect local and long-range lexicalsemantic networks. Brain and Language, 127(3), 440-451.

Mitra, P. P., \& Pesaran, B. (1999). Analysis of dynamic brain imaging data. Biophysical Journal, 76(2), 691-708.

Obleser, J., \& Kotz, S. a. (2010). Expectancy constraints in degraded speech modulate the language comprehension network. Cerebral Cortex, 20(3), 633-640.

Obleser, J., \& Kotz, S. a. (2011). Multiple brain signatures of integration in the comprehension of degraded speech. Neurolmage, 55(2), 713-723.

Obleser, J., \& Weisz, N. (2012). Suppressed alpha oscillations predict intelligibility of speech and its acoustic details. Cerebral Cortex, 22(11), 2466-2477.

Obleser, J., Wöstmann, M., Hellbernd, N., Wilsch, A., \& Maess, B. (2012). Adverse listening conditions and memory load drive a common $\alpha$ oscillatory network. Journal of Neuroscience, 32(36), 12376-12383.

Oostenveld, R., Fries, P., Maris, E., \& Schoffelen, J.-M. (2011). FieldTrip: Open source software for advanced analysis of MEG, EEG, and invasive electrophysiological data. Computational Intelligence and Neuroscience, 2011, 156869.

Osipova, D., Hermes, D., \& Jensen, O. (2008). Gamma power is phase-locked to posterior alpha activity. PloS One, 3(12), e3990.

Otsuka, S., \& Kawaguchi, J. (2007). Divided attention modulates semantic activation: Evidence from a nonletter-level prime task. Memory \& Cognition, 35(8), 2001-2011.

Peña, M., \& Melloni, L. (2012). Brain oscillations during spoken sentence processing. Journal of Cognitive Neuroscience, 24(5), 1149-1164.

Pluymaekers, M., Ernestus, M., \& Baayen, R. H. (2005). Lexical frequency and acoustic reduction in spoken Dutch. The Journal of the Acoustical Society of America, 118(4), 2561.

Ranbom, L. J., \& Connine, C. M. (2007). Lexical representation of phonological variation in spoken word recognition. Journal of Memory and Language, 57(2), 273-298.

Schuppler, B., Ernestus, M., Scharenborg, O., \& Boves, L. (2011). Acoustic reduction in conversational Dutch: A quantitative analysis based on automatically generated segmental transcriptions. Journal of Phonetics, 39(1), 96-109.

Shahin, A. J., Picton, T. W., \& Miller, L. M. (2009). Brain oscillations during semantic evaluation of speech. Brain and Cognition, 70(3), 259-266.

Smith, M. S., Bentin, S., \& Spalek, T. M. (2001). Attention constraints of semantic activation during visual word recognition. Journal of Experimental Psychology: Learning, Memory and Cognition, 27(5), 1289-1298.

Strauß, A., Kotz, S. a., Scharinger, M., \& Obleser, J. (2014). Alpha and theta brain oscillations index dissociable processes in spoken word recognition. Neurolmage, 97C, 387-395.

Strauß, A., Wostmann, M., \& Obleser, J. (2014). Cortical alpha oscillations as a tool for auditory selective inhibition. Frontiers in Human Neuroscience, 8, 1-7.

Tucker, B. V., \& Warner, N. (2007). Inhibition of processing due to reduction of the American English flap. In Proceedings of the 16th international congress of phonetic sciences, Saarbrücken (pp. 1949-1952).

Van Bergem, D. R. (1994). A model of coarticulatory effects on the schwa. Speech Communication, 14(2), 143-162.

Van de Ven, M., Ernestus, M., \& Schreuder, R. (2012). Predicting acoustically reduced words in spontaneous speech: The role of semantic/syntactic and acoustic cues in context. Laboratory Phonology, 3(2), 455-481.

Van de Ven, M., Tucker, B. V., \& Ernestus, M. (2011). Semantic context effects in the comprehension of reduced pronunciation variants. Memory E Cognition, 39(7), 1301-1316.

Varela, F., Lachaux, J. P., Rodriguez, E., \& Martinerie, J. (2001). The brainweb: Phase synchronization and large-scale integration. Nature Reviews Neuroscience, 2(4), 229-239.

Wang, L., Zhu, Z., \& Bastiaansen, M. (2012). Integration or predictability? A further specification of the functional role of gamma oscillations in language comprehension. Frontiers in Psychology, 3(June), 187.

Weisz, N., Hartmann, T., Müller, N., Lorenz, I., \& Obleser, J. (2011). Alpha rhythms in audition: Cognitive and clinical perspectives. Frontiers in Psychology, 2, 73.

Wilsch, A., Henry, M. J., Herrmann, B., Maess, B., \& Obleser, J. (2014). Alpha oscillatory dynamics index temporal expectation benefits in working memory. Cerebral Cortex, 1-9. 